\title{
encephalitis
}

\section{Changes in laboratory mice after observation of deceased conspecifics: a pilot suicidality study in animals}

\author{
Daejong Jeon ${ }^{1, *}$, Sangwoo Kim ${ }^{2, *}$, Sang Kun $\mathrm{Lee}^{2}$, Kon Chu ${ }^{2}$ \\ ${ }^{1}$ Advanced Neural Technologies, Co., Seoul, Korea \\ ${ }^{2}$ Laboratory for Neurotherapeutics, Department of Neurology, Biomedical Research Institute, Seoul National University Hospital, Seoul, \\ Korea
}

\section{Purpose}

Suicidality can be a serious feature of psychiatric symptoms in encephalitis. Investigating the psychiatric behavior associated with suicidality in animal models of encephalitis is important; thus, determining whether normal laboratory animals are aware of death is necessary.

\section{Methods}

To examine the behavioral and brain activity changes associated with death of conspecifics, laboratory mice were exposed to a cadaveric mouse or an anesthetized mouse. Behavioral tasks associated with anxiety and locomotion were conducted after repeated exposure. Neural activity in the medial prefrontal cortex during the cadaver exploration was investigated using electroencephalographic recordings.

\section{Results}

During repeated exposure, mice in the cadaver group showed a gradual decrease in time exploring the cadaver, which was not observed in mice in the anesthesia group. The cadaver group also exhibited increased levels of anxiety in the light/dark transition and elevated plus maze tasks and displayed increased locomotor activity in the open field test. In an electrophysiological study, different brain oscillations were observed when mice were exposed to a cadaveric mouse and an anesthetized mouse. Enhanced delta-band activity and reduced theta- and alpha-band activities were observed during cadaver exploration.

\section{Conclusion}

The present study results showed that experiences involving dead conspecifics strongly affect mouse behavior and brain activity. These findings may be helpful in treating patients with psychiatric symptoms and aid in understanding the concept of death recognition/awareness in laboratory animals.

Keywords: Encephalitis, Suicidality, Laboratory mice, Psychiatric behaviors, Electroencephalography

Received: May 20, 2021 Revised: June 7, 2021 Accepted: June 11, 2021

Correspondence: Sang Kun Lee

Department of Neurology, Seoul National University Hospital, 101 Daehak-ro, Jongno-gu, Seoul 03080, Korea

E-mail: sangkun2923@gmail.com

ORCID: https://orcid.org/0000-0003-1908-0699

Kon Chu

Department of Neurology, Seoul National University Hospital, 101 Daehak-ro, Jongno-gu, Seoul 03080, Korea

E-mail: stemcell.snu@gmail.com

ORCID: https://orcid.org/0000-0001-5863-0302

*These authors contributed equally to this study as co-first authors.

Copyright (C) 2021 by The Korean Encephalitis and Neuroinflammation Society

This is an open access article distributed under the terms of the Creative Commons Attribution Non-Commercial License (http://creativecommons.org/licenses/by-nc/4.0/) which permits unrestricted non-commercial use, distribution, and reproduction in any medium, provided the original work is properly cited. 


\section{Introduction}

Encephalitis can often be accompanied by emotional and psychological problems, such as anxiety, depression, and mood swings. In antibody-mediated autoimmune encephalitis, such as anti- $N$-methyl-D-aspartate receptor encephalitis, the degree of psychiatric symptoms is severe. In addition, suicidality can be a serious feature of psychiatric symptoms in encephalitis [1]. Therefore, studying suicidality in animals can be helpful in treating patients with psychiatric brain disorders.

Many nonhuman mammalian species show unique responses to dead conspecifics, such as remaining next to the deceased conspecific, vocalizations, grooming, licking, or carrying the body [2-6]. To date, whether these behavioral observations are relevant to human behaviors regarding death, including death-related psychological states (e.g., grief, mourning, bereavement, and lamentation) and fear or anxiety of mortality remains unclear. However, animals presumably exhibit such behaviors because they can distinguish between life and death and recognize death as something other than living [3,7-10].

In the wild, animals may occasionally observe dying conspecifics or may encounter dead conspecifics due to events such as aging, disease, or enemy attack. This experience may be traumatic as the animals come to recognize or become aware of death. However, animals that live in a laboratory cannot have such observational experiences involving dead bodies of their conspecifics owing to animal welfare practices and the control of experiments in accordance with the guidelines of the Institutional Animal Care and Use Committee [11]. Thus, whether laboratory animals can discriminate between living and dead conspecifics, and between life and death, remains unclear. If laboratory animals can process this distinction, their responses and the importance of these experiences remain unclear. Answers to these questions may provide basic information regarding animal thanatology and for suicidality studies in animals as well as help elucidate the evolutionary origins of death-related behaviors.

Mice are commonly used experimental animals. They are highly social and even display empathy-like behaviors toward each other [12-15]. Thus, mouse models can be useful for assessing the neurobiological correlates of death recognition. In the present pilot suicidality study, laboratory animals were used to determine how a dead body (cadaver) of a conspecific affected mouse behavior and behavioral changes were compared with those elicited using anesthetized mice. Furthermore, neural activity in the medial prefrontal cortex (mPFC) during cadaver ex- ploration was investigated using electrophysiological assessment in vivo.

\section{Methods}

\section{Animals and treatments}

Male C57BL/6 mice were used in the present study. Animals were maintained with free access to food and water under a 12hour light/dark cycle. All experiments were approved by the Institutional Animal Care and Use Committee in Seoul National University Hospital (SNUH-IACUC, No. 14-0210-S1A1) and animals were maintained in the facility accredited AAALAC International (\#001169) in accordance with Guide for the Care and Use of Laboratory Animals 8th edition (National Research Council 2011). All efforts were made to minimize suffering. The overall study consisted of two major experiments shown in Figure 1A. Mice 10-12 weeks old were used as subject mice and divided into two groups: (1) cadaver group, exposed to a cadaveric conspecific; (2) anesthesia group, exposed to an anesthetized conspecific. Age-matched male C57BL/6 mice that had been used for other behavioral experiments and were scheduled to be sacrificed were used as cadaveric or anesthetized mice. The mice scheduled to be used as cadaveric mice were sacrificed using $\mathrm{CO}_{2}$ immediately prior to each experiment. The mice scheduled to be used as anesthetized mice were deeply anesthetized with an intraperitoneal injection of ketamine (70 $\mathrm{mg} / \mathrm{kg}$ ) and xylazine hydrochloride $(10 \mathrm{mg} / \mathrm{kg})$ immediately prior to each experiment.

\section{Social investigation of a cadaver and an anesthetized mouse target}

The experiment was performed as previously described [16]. A single subject mouse was allowed to roam freely in a clean cage $(22 \times 19 \times 17 \mathrm{~cm}, 3-\mathrm{cm}$ sawdust layer) for 10 minutes. The cages used were identical to cages in which the mice were normally housed. After the 10-minute period, a cadaveric or anesthetized male mouse (target mouse) was placed in the middle of the cage. Then, the subject mouse was allowed to roam freely for 15 minutes (test session). Behavior toward the target mouse was video-recorded for 15 minutes and the duration of contact (sniffing, touching, and climbing) were measured and analyzed for 5 or 10 minutes. A mouse was considered to be sniffing the target mouse when its head was facing the target mouse within 1 inch. The cage, target mouse, and sawdust were changed for every subject mouse. This experiment was repeatedly performed once a day for 3 days. 
Figure 1 Experimental design and social investigation of a cadaver
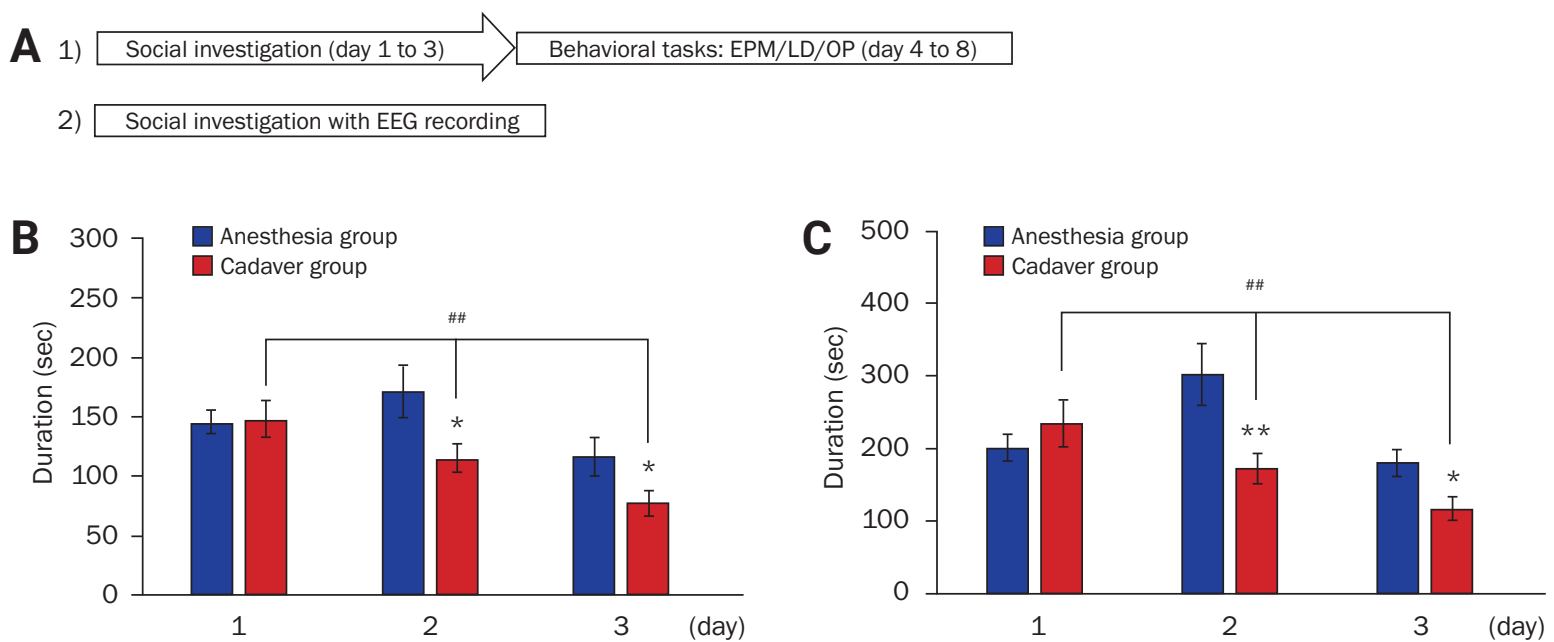

(A) Simple schematic for the experiments: 1) Behavioral tasks after the social investigation of a cadaveric mouse target or an anesthetized mouse target for 3 days; 2) electroencephalographic (EEG) recordings during the social investigation. (B, C) In the 3-day social investigation of a cadaveric mouse target or an anesthetized mouse target, the contact duration was assessed for the first 5 minutes (B) and 10 minutes (C) on each day. The duration of contact in the cadaver group, but not in the anesthesia group, continued to decrease for 3 days, and the cadaver group showed reduced duration of contact compared with the anesthesia group.

EPM, elevated plus maze; LD, light/dark transition; OP, open field.

Comparison between the two groups: ${ }^{*} p<0.05,{ }^{*} \mathrm{p}<0.01$; Student t-test. Comparison within each group: ${ }^{\#} \mathrm{p}<0.01$; one-way analysis of variance.

\section{Elevated plus maze and light/dark transition}

For assessment of anxiety, elevated plus maze and light/dark transition tasks were performed as previously described $[13,16,17]$. The elevated plus maze was made of plastic and consisted of two white open arms $(25 \times 8 \mathrm{~cm})$, two black enclosed arms $(25 \times 8 \times 20 \mathrm{~cm})$, and a central platform $(8 \times 8 \times 8 \mathrm{~cm})$ in the form of a cross. The maze was placed $50 \mathrm{~cm}$ above the floor. Mice were individually placed in the center with their heads directed toward one of the closed arms. The total time spent in each arm or in the center was analyzed by video monitoring for 5 minutes. An arm entry was defined and counted when all four paws crossed from the center into an arm and was used for measuring the amount of time spent in each arm.

For the light/dark transition task, a light/dark box $(30 \times 45 \times 27$ $\mathrm{cm}$ ) made of plastic comprised of a dark compartment (one third of the total area) and light compartment with a hole in the middle. The light compartment was illuminated at 400 lux. The elapsed time to entry (all four paws) into the light compartment (latency) and the amount of time (duration) spent in each compartment were measured over a 5-minute period by video monitoring.

\section{Open field test}

To assess locomotor activity, the open field test was performed as previously described $[13,18]$. The open field box was made of white plastic $(40 \times 40 \times 40 \mathrm{~cm})$. Individual mice were placed in the periphery of the field and the paths of the animals recorded with a video camera. The total distance traveled for 10 minutes was analyzed using a software program (EthoVision XT; Noldus Information Technology, Wageningen, The Netherlands).

\section{In vivo electrophysiology for electroencephalographic} Electroencephalographic (EEG) surgery and recording in vivo were performed as previously described $[13,18,19]$. For EEG monitoring during a social behavioral task, nine naïve mice were subjected to EEG surgery 1 week before the experiment. For surgery, the animals were anesthetized with an intraperitoneal injection of ketamine (90 $\mathrm{mg} / \mathrm{kg}$ ) and xylazine hydrochloride $(40 \mathrm{mg} / \mathrm{kg})$. Electrode implantation was performed using a stereotaxic apparatus (Kopf Instruments, Tujunga, CA, USA). EEG recordings were obtained with tungsten electrodes $(0.005$ in, $2 \mathrm{M} \Omega$ ), positioned in the $\mathrm{mPFC}$ (anterior-posterior, -1.94 to -1.70 mm; medial-lateral, 0.25-0.45 $\mathrm{mm}$; and dorsal-ventral, $2.75 \mathrm{~mm}$ ) from the bregma with grounding over the cerebellum. The electrodes were fixed to the skull with cyanoacrylate adhesive and dental acrylic cement. After a 1-week recovery from brain surgery, animals were placed in a small acrylic cage (15 $\times$ $20 \times 15 \mathrm{~cm}$ ) and allowed to move freely in the cage. EEG recordings combined with video monitoring were performed simultaneously during the behavioral task on social investigation of a 
cadaveric conspecific or an anesthetized conspecific. Subject mice were sequentially exposed to anesthetized or cadaveric conspecifics at 15-minute intervals. The order of exposure to targets was random. Two epochs (10 seconds each) of EEG signals during exploration of the conspecific by each mouse, from 1 to 3 minutes after the start of the behavioral task, were randomly selected and used for analysis. The five EEG frequency bands, delta (1.5-4 Hz), theta (4-8 Hz), alpha (8-12 Hz), beta $(12-30 \mathrm{~Hz})$, and gamma $(30-60 \mathrm{~Hz})$, were analyzed for EEG power. The relative normalized power at each individual frequency was presented as a fraction of the sum of powers at all frequencies. The electrical activities were recorded after amplification $(\times 12,000)$, bandpass filtering from 0.1 to $100 \mathrm{~Hz}$, and digitization at a $400 \mathrm{~Hz}$ sampling rate (AS 40) with a digital electroencephalography system (Comet XL; Astro-Med, Inc., Warwick, RI, USA). EEG-video data obtained were analyzed offline using PSG Twin (Astro-Med) and Clampfit (Axon Instruments, Foster City, CA, USA).

\section{Statistical analysis}

All data are presented as means \pm standard error of the mean. Analysis of variance (ANOVA) was used to conduct multiple comparisons of means. Student t-test was performed to determine statistical differences between two means. A p-value < 0.05 was considered statistically significant.

\section{Results}

\section{Reduced social investigation of cadaver}

To determine how laboratory mice are affected by a dead conspecific, mice were first allowed to explore a dead conspecific (cadaver). The social investigative activity was assessed as duration of contact with the target (a cadaver or an anesthetized mouse). On the first day, the cadaver (exposed to a cadaveric conspecific) $(\mathrm{n}=17 ; 147.88 \pm 15.65$ seconds over 5 minutes, Figure 1B; $232.93 \pm 33.04$ seconds over 10 minutes, Figure $1 \mathrm{C}$ ) and anesthesia groups (exposed to an anesthetized conspecific) ( $\mathrm{n}=13 ; 145.32 \pm 9.56$ seconds over 5 minutes, Figure 1B; 199.45 \pm 19.56 seconds over 10 minutes, Figure 1C) spent a similar amount of time in contact with their targets. However, on the second day, the cadaver group $(114.72 \pm 11.96$ seconds over 5 minutes, Figure 1B; $170.08 \pm 21.46$ seconds over 10 minutes, Figure 1C) showed a significantly shorter contact duration than the anesthesia group $(170.85 \pm 21.89$ seconds over 5 minutes, $p$ $<0.05$, Student t-test, Figure 1B; $301.20 \pm 42.81$ seconds over 10 minutes, $\mathrm{p}<0.01$, Student t-test, Figure 1C). Similarly, on the third day, the cadaver group (76.92 \pm 10.78 seconds over 5 minutes, Figure 1B; $114.85 \pm 15.60$ seconds over 10 minutes, Figure 1C) showed a significantly shorter contact duration than the anesthesia group (116.25 \pm 15.52 seconds over 5 minutes, $\mathrm{p}<0.05$, Student t-test; Figure 1B; $178.78 \pm 19.15$ seconds over $10 \mathrm{~min}$ utes, $\mathrm{p}<0.05$, Student t-test; Figure 1C). Notably, the contact duration in the cadaver group gradually became shorter from day 1 to day $3\left(\mathrm{~F}_{2,48}=7.50, \mathrm{p}<0.01\right.$, one-way ANOVA, Figure $1 \mathrm{~B} ; \mathrm{F}_{2,48}$ $=5.83, \mathrm{p}<0.01$, one-way ANOVA, Figure $1 \mathrm{C}$ ); however, the anesthesia group showed no difference $\left(\mathrm{F}_{2,36}=2.85, \mathrm{p}=0.07\right.$, oneway ANOVA; Figure $1 \mathrm{~B})$ or increase on day $2\left(\mathrm{~F}_{2,36}=4.85, \mathrm{p}<\right.$ 0.05, one-way ANOVA; Figure 1C). The results indicated that exploratory activities differed depending on social targets and mice respond to a dead conspecific in a manner different from their response to a living anesthetized conspecific.

\section{Cadaver group showed increased anxiety levels and locomotor activity}

After the 3-day exposure experiment, the cadaver and anesthesia groups were subjected to elevated plus maze, light/dark transition, and open field tests to assess anxiety and locomotion. In the elevated plus maze task (Figure 2A), both the cadaver ( $\mathrm{n}$ $=17,10.00 \pm 2.78$ seconds $)$ and anesthesia groups $(\mathrm{n}=13,7.35$ \pm 2.49 seconds) spent less time in the open arms than the control group $\left(\mathrm{n}=17,24.62 \pm 3.02\right.$ seconds, $\mathrm{F}_{2,43}=11.10, \mathrm{p}<$ 0.001 , one-way ANOVA). In addition, the cadaver (251.96 \pm 6.54 seconds) and anesthesia groups (261.72 \pm 4.56 seconds) spent more time in the closed arms than the control group (229.84 \pm 5.91 seconds, $\mathrm{F}_{2,43}=7.67, \mathrm{p}<0.01$, one-way ANOVA). Significant differences were not observed between the cadaver and anesthesia groups.

In the light/dark transition task (Figure 2B), the cadaver group (n $=16,165.80 \pm 25.10$ seconds) displayed a longer latency in the light compartment compared with the control group $(n=17$, $93.09 \pm 16.74$ seconds, $\mathrm{p}<0.05$, Student t-test). Furthermore, the cadaver group displayed longer and shorter amounts of time in the dark (cadaver, $257.93 \pm 10.78$ seconds; control, $226.03 \pm$ 7.64 seconds, $\mathrm{p}<0.05$, Student t-test) and light compartments (cadaver, $42.07 \pm 10.38$ seconds; control, $73.97 \pm 7.64$ seconds, $\mathrm{p}<0.05$, Student t-test), respectively, compared with the control group. However, the anesthesia group spent a similar amount of time in each compartment compared with the control group.

To assess locomotor activity, an open field test was performed. Mice in the cadaver group traveled a greater distance $(n=15$, $3,758.78 \pm 106.85 \mathrm{~cm})$ than mice in the control group $(\mathrm{n}=17$, 3,323.42 $\pm 95.95 \mathrm{~cm}, \mathrm{p}<0.01$, Student t-test). However, mice in the anesthesia group $(\mathrm{n}=13,3,395.06 \pm 90.06 \mathrm{~cm})$ traveled a distance similar to mice in the control group. 
Figure 2 Behavioral alterations in mice exposed to a cadaver

A

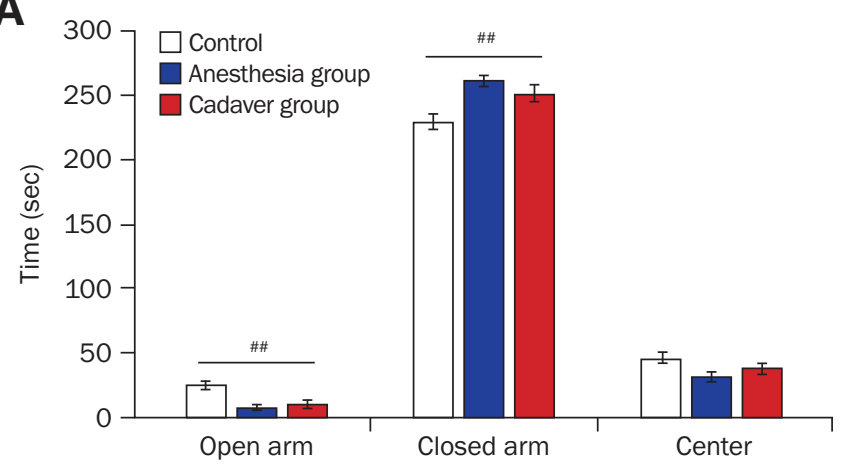

B

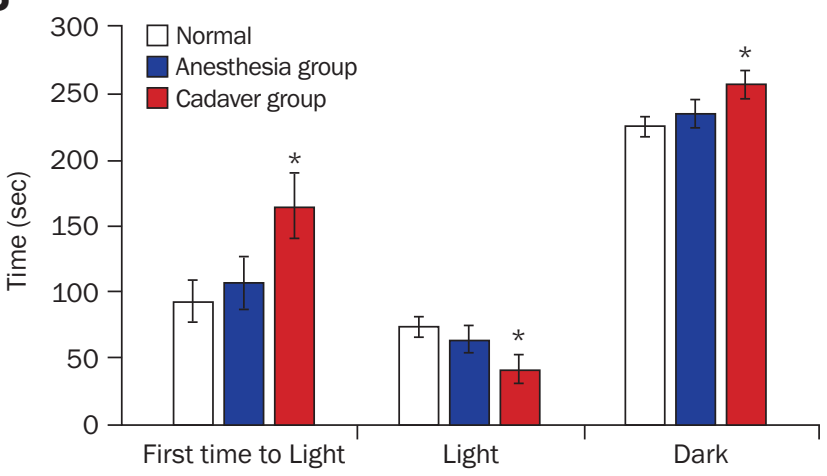

C

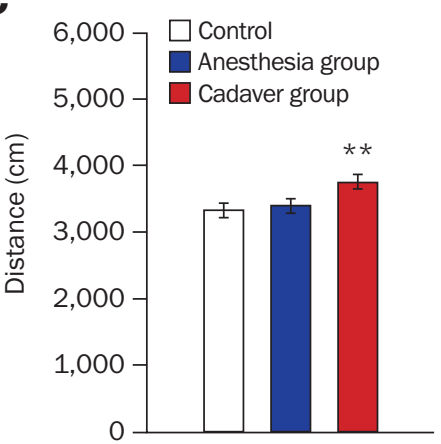

(A) Elevated plus maze task: the cadaver and anesthesia groups spent less and more time in the open and closed arms, respectively, than the control group. There was no difference between the cadaver and the anesthesia groups. Comparison among groups: ${ }^{\#} p<0.01$; one-way analysis of variance. (B) Light/dark transition task: the cadaver group, but not the anesthesia group, showed a longer latency to enter the light compartment, spent less time in the light compartment, and spent more time in the dark compartment than the control group. There was no difference between the control and the anesthesia groups. Comparison with control group: ${ }^{*} p<0.05$; Student t-test. (C) Open field test: the cadaver group, but not the anesthesia group, exhibited an increase in total distance traveled in the open field box compared with the control group. There was no difference between the control and anesthesia groups. Comparison with control or anesthesia group: ** $p<0.01$; Student t-test.

Taken together, these results demonstrated that mice exposed to a cadaveric conspecific had increased levels of anxiety and locomotor activity compared with naïve control mice or mice ex- posed to an anesthetized conspecific.

\section{Differences in brain oscillations in the medial} prefrontal cortex during exploration of cadaveric and anesthetized mice

The mPFC has an important role in social cognitive behavior, including novelty detection and fear recognition [20-26]. EEG signals were measured from the mPFC while mice investigated cadaveric and anesthetized conspecifics (Figure 3A) and brain oscillations compared using a frequency-wise power spectrum analysis. During exploration of a cadaver, subject mice showed significantly enhanced delta-band activity and reduced thetaand alpha-band activities in the MPFC relative to their activities during exploration of an anesthetized conspecific ( $p<0.05$, Student t-test; Figure 3B). Normalized power spectrum analysis of another EEG signal epoch also showed a similar result (Figure 3C). The results indicated that mice showed different brain activities toward cadaveric and anesthetized conspecifics and can distinguish live from dead bodies.

\section{Discussion}

In the wild, animals may frequently encounter dead bodies and observe the death of their family members. These experiences, together with education from family or social groups, may help animals understand or become aware of death and may cause them to exhibit specific behavioral responses to the dead (or to react differently to dead bodies), such as remaining near or carrying the deceased conspecific, as well as vocalizations, grooming, or licking [2-6].

Death-related behavior in primates has been reported in several studies [27-31]. For example, a daughter chimpanzee was reported to groom and stay with her mother's body, and to sleep fitfully, for a few days after her mother's death [32]. In addition, elephants reportedly have a generalized response to a dead body or death [33-35]. Elephants gather around a dead conspecific, manipulate the body with their feet and trunks, and often stand vigil for several days, which may indicate their awareness of death [33]. Numerous reports have been published of death-related behavior in other animals, such as giraffes [36], dogs [37], and whales or dolphins [38,39]. Thus, animals, specifically social mammalian species, can distinguish between life and death; however, what a dead body signifies to these animals is unknown.

In the present study, the effects of a dead body on a laboratory mouse that has never previously encountered a dead conspecific and likely has not received any education regarding death 
Figure 3 Differences in brain oscillations of mice exposed to a cadaveric mouse target and to an anesthetized mouse target

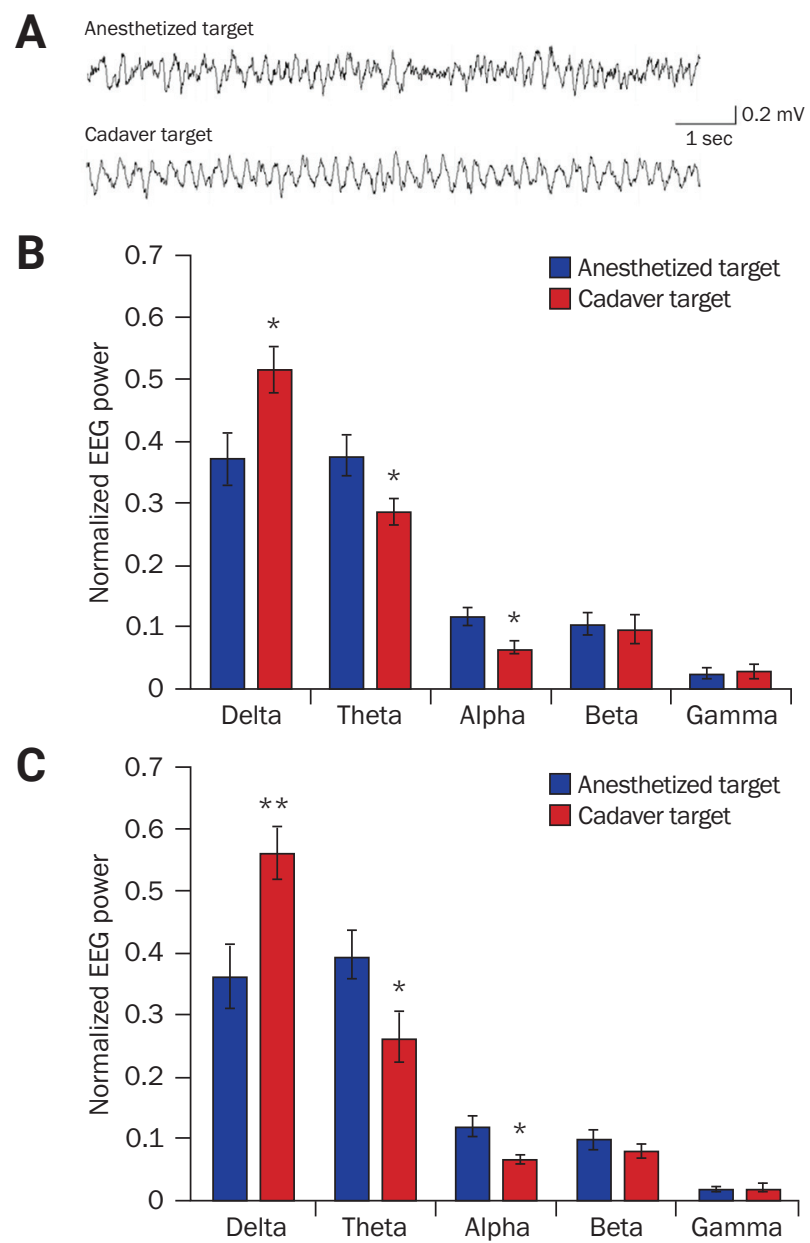

(A) Sample traces of electroencephalographic (EEG) signals from mouse medial prefrontal cortex neurons during investigations of the cadaveric and anesthetized mouse targets. (B, C) Power spectra analyses of two epochs of EEG signal: power spectra analyses revealed that mice showed increased delta-band activity and reduced theta- and alpha-band activities when they explored a cadaveric mouse target than when they explored an anesthetized mouse target.

Comparison between the two targets in each band: ${ }^{*} p<0.05, * * p<0.01$; Student t-test.

from its family (parents or cagemates) were investigated. Exposure to a cadaveric conspecific rendered mice more anxious than exposure to an anesthetized conspecific. In the social investigation experiment, differences were not observed in contact time between the cadaver and anesthesia groups on the first day; however, differences were observed on the second and third days. If mice had an innate fear of dead bodies, mice exposed to a cadaveric conspecific should have exhibited reduced contact time compared with mice exposed to an anesthetized conspecific on the first day. The presence or smell of a predator provokes immediate innate fear in rodents such as rats and mice. Mice avoid the location of the predator, even when they have never been exposed to it and have no prior learning experience [40]. However, on the second and third days, mice exposed to the cadaveric conspecific displayed reduced contact duration compared with mice exposed to an anesthetized conspecific. Thus, initially the dead body was likely a novelty, and source of curiosity, rather than a source of innate fear. Unlike exposure to an anesthetized mouse, the odor from a dead body can cause increased level of anxiety and locomotion and reduced contact duration.

Although difference was not observed in the contact duration between the two groups on the first day, mice might have suspected a problem with the cadaver and acquired some information regarding the cadaver during the first investigation; they exhibited different neural activities in the MPFC when investigating a cadaveric conspecific or an anesthetized conspecific in the electrophysiological experiment. The mice may have learned to fear or experienced anxiety from repeated exposure to cadaveric conspecifics on the second and third days. However, the mice may simply have lost interest more quickly in a body that was dead compared with an alive but anesthetized body. In addition, whether the fear of a cadaveric conspecific in this study included fear of mortality could not be determined [41,42]. Further experiments are needed to determine whether exposure to a cadaveric conspecific or exposure to potential death is recognized as a fearful situation or source of anxiety in mice. In addition, further studies using familiar mice, such as cagemates or siblings, as cadavers may significantly advance the understanding of the neural basis of death-related behavior in animals.

In the present study, several experiments were performed to determine how exposure to a cadaveric conspecific affects laboratory mice and what relevance it has. Notably, exposure to the cadaveric conspecific rendered laboratory mice fearful or anxious, and changed their brain activities. Encountering the dead body of a conspecific may be a traumatic experience for laboratory mice and they may acquire fear of a cadaver without experiencing an innate fear of death. Finally, the results indicated that laboratory mice can learn about death through fear or anxiety induced by exposure to a cadaveric conspecific. Although whether laboratory mice learned or became aware of death through investigation of a cadaver conspecific is unknown, experiments using animal cadaveric conspecifics may provide information and facilitate further studies of brain disorders such as suicidality and psychosocial behaviors. 


\section{Conflicts of Interest}

Daejong Jeon has been an associate editor of $\boldsymbol{e n c e p h a l i t i s ~ s i n c e ~}$ October 2020. Sang Kun Lee and Kon Chu have been on the editorial board of encephalitis since October 2020. They were not involved in the review process of this original article. No other potential conflict of interest relevant to this article was reported.

\section{Author Contributions}

Conceptualization, Methodology: Jeon D, Lee SK, Chu K; Investigation, Data curation, Formal analysis: Jeon D, Kim S; Resources: Lee SK, Chu K; Funding acquisition: Jeon D, Lee SK, Chu K; Writing-original draft: Jeon D; Writing-review and editing: all authors.

\section{Acknowledgments}

We would like to thank Ah Reum Yang (Laboratory for Neurotherapeutics, Department of Neurology, Biomedical Research Institute, Seoul National University Hospital, Seoul, Korea) for her help in the behavioral tests. This work was supported by Korea Health $21 \mathrm{R} \& \mathrm{D}$ grants (HI12C0035) funded by Ministry of Health and Welfare and supported by a grant from Advanced Neural Technologies (0620182930).

\section{References}

1. Zhang L, Sander JW, Zhang L, et al. Suicidality is a common and serious feature of anti-N-methyl-D-aspartate receptor encephalitis. J Neurol 2017;264:2378-2386.

2. King BJ. When animals mourn. Sci Am 2013;309:62-67.

3. Pierce J. The dying animal. J Bioeth Inq 2013;10:469-478.

4. King BJ. Animal mourning: précis of how animals grieve (King 2013). Anim Sentience 2016;1:1.

5. King BJ. How animals grieve. Chicago: University of Chicago Press; 2013.

6. Angier N. Do animals grieve over death like we do? [Internet]. The New York Times; 2008 Sep 2 [cited 2021 May 20]. Available from: https://www.nytimes.com/2008/09/02/health/02iht-02angi.15827535. html.

7. Alderton D. Animal grief: how animals mourn. Dorchester, UK: Veloce Publishing Ltd; 2011.

8. Brooks Pribac T. Animal grief. Anim Stud J 2013;2:67-90.

9. DeGrazia D. Self-awareness in animals: the philosophy of animal minds. Cambridge, UK: Cambridge University Press; 2009.

10. Peña-Guzmán DM. Can nonhuman animals commit suicide? Anim Sentience 2017;2:1.
11. Silverman J, Suckow MA, Murthy S. The IACUC handbook. Boca Raton (FL): CRC Press; 2014.

12. Chen Q, Panksepp JB, Lahvis GP. Empathy is moderated by genetic background in mice. PLoS One 2009;4:e4387.

13. Jeon D, Kim S, Chetana M, et al. Observational fear learning involves affective pain system and Cav1.2 Ca2+ channels in ACC. Nat Neurosci 2010;13:482-488.

14. Keum S, Shin HS. Rodent models for studying empathy. Neurobiol Learn Mem 2016;135:22-26.

15. Pisansky MT, Hanson LR, Gottesman II, Gewirtz JC. Oxytocin enhances observational fear in mice. Nat Commun 2017;8:2102.

16. Jang Y, Lee SH, Lee B, et al. TRPM2, a susceptibility gene for bipolar disorder, regulates glycogen synthase kinase-3 activity in the brain. J Neurosci 2015;35:11811-11823.

17. Takao K, Miyakawa T. Light/dark transition test for mice. J Vis Exp 2006;(1):104.

18. Khalid A, Kim BS, Seo BA, et al. Gamma oscillation in functional brain networks is involved in the spontaneous remission of depressive behavior induced by chronic restraint stress in mice. BMC Neurosci 2016;17:4.

19. Khalid A, Kim BS, Chung MK, Ye JC, Jeon D. Tracing the evolution of multi-scale functional networks in a mouse model of depression using persistent brain network homology. Neuroimage 2014;101:351-363.

20. Bicks LK, Koike H, Akbarian S, Morishita H. Prefrontal cortex and social cognition in mouse and man. Front Psychol 2015;6:1805.

21. Courtin J, Bienvenu TC, Einarsson EÖ, Herry C. Medial prefrontal cortex neuronal circuits in fear behavior. Neuroscience 2013;240:219-242.

22. Daffner KR, Mesulam MM, Scinto LF, et al. The central role of the prefrontal cortex in directing attention to novel events. Brain 2000;123(Pt 5):927-939

23. Dias R, Honey RC. Involvement of the rat medial prefrontal cortex in novelty detection. Behav Neurosci 2002;116:498-503.

24. Ko J. Neuroanatomical substrates of rodent social behavior: the medial prefrontal cortex and its projection patterns. Front Neural Circuits 2017;11:41

25. Nakao T, Matsumoto T, Shimizu D, et al. Resting state low-frequency fluctuations in prefrontal cortex reflect degrees of harm avoidance and novelty seeking: an exploratory NIRS study. Front Syst Neurosci 2013;7:115

26. Yochiy A, Britto LR, Hunziker MH. Novelty, but not operant aversive learning, enhances Fos and Egr-1 expression in the medial prefrontal cortex and hippocampal areas of rats. Behav Neurosci 2012;126:826834.

27. Morin R. A conversation with Koko the gorilla [Internet]. Boston: The Atlantic; 2015 Aug 28 [cited 2021 May 20]. Available from: https:// www.theatlantic.com/technology/archive/2015/08/koko-the-talkinggorilla-sign-language-francine-patterson/402307/. 
28. Goodall J. My life with the chimpanzees. New York: Simon \& Schuster; 1996.

29. Cronin KA, van Leeuwen EJ, Mulenga IC, Bodamer MD. Behavioral response of a chimpanzee mother toward her dead infant. Am J Primatol 2011;73:415-421.

30. Biro D, Humle T, Koops K, Sousa C, Hayashi M, Matsuzawa T. Chimpanzee mothers at Bossou, Guinea carry the mummified remains of their dead infants. Curr Biol 2010;20:R351-R352.

31. Anderson JR. A primatological perspective on death. Am J Primatol 2011;73:410-414.

32. Anderson JR, Gillies A, Lock LC. Pan thanatology. Curr Biol 2010;20: R349-R351.

33. Douglas-Hamilton I, Bhalla S, Wittemyer G, Vollrath F. Behavioural reactions of elephants towards a dying and deceased matriarch. Appl Anim Behav Sci 2006;100:87-102.

34. Goldman J. Death rituals in the animal kingdom [Internet]. BBC News; 2012 Sep 19 [cited 2021 May 20]. Available from: https://www. bbc.com/future/article/20120919-respect-the-dead.

35. McComb K, Baker L, Moss C. African elephants show high levels of interest in the skulls and ivory of their own species. Biol Lett 2006;2:2628.

36. Bercovitch FB. Giraffe cow reaction to the death of her newborn calf. Afr J Ecol 2013;51:376-379.

37. Fox M. Dog body, dog mind: Exploring canine consciousness and total well-being. Lanham: Rowman \& Littlefield; 2007.

38. Bearzi G, Eddy L, Piwetz S, Reggente MA, Cozzi B. Cetacean behavior toward the dead and dying. Encycl Anim Cogn Behav 2017;1:1-30.

39. Reggente MA, Papale E, McGinty N, Eddy L, de Lucia GA, Bertulli CG. Social relationships and death-related behaviour in aquatic mammals: a systematic review. Philos Trans R Soc Lond B Biol Sci 2018; 373:20170260.

40. Dielenberg RA, McGregor IS. Habituation of the hiding response to cat odor in rats (Rattus norvegicus). J Comp Psychol 1999;113:376-387.

41. Moor A. Awareness of death: a controllable process or a traumatic experience? Folk Electron J Folk 2002;(20-22):92-114.

42. San Filippo D. Perspectives on the fears of death \& dying. Chicago: Faculty Publications, National Louis University; 2006. 